\title{
NOTAS
}

\section{PARA LA HISTORIA DE CAMISETA, UN AMERICANISMO INADVERTIDO}

\begin{abstract}
Al estudiar el léxico hispánico de la Relación de Michoacán llamó mi atención el uso de la palabra camiseta en esta temprana crónica de Indias, escrita hacia 1541 y cuyo manuscrito se encuentra depositado en la Real Biblioteca del Monasterio de El Escorial ${ }^{1}$. Quizá por ser una voz frecuente en el español actual y por carecer del sentido novedoso o exótico característico de las primeras voces americanas, me sorprendió que camiseta apareciera en ese texto de una manera que mi intuición identificó inmediatamente con un americanismo ${ }^{2}$, debido a que su modo de funcionamiento me recordó al utilizado por los cronistas para introducir las nuevas palabras americanas ${ }^{3}$.

En este trabajo pretendo mostrar que camiseta es una creación léxica americana, que se documenta desde el siglo xvI hasta la segunda mitad del siglo XIX sólo en el español de América con el significado de 'camisa o vestido de indio'. Hasta la mitad del siglo xix el derivado de la palabra patrimonial camisa no aparece con un sentido de 'prenda de vestir' en el español de España, momento en el que también se documenta en el de América, pero donde ya no refiere a la indumentaria indígena. Desde entonces camiseta adquiere una mayor
\end{abstract}

\footnotetext{
1 Relaçión delas çerimonias y rritos y poblaçión y gobernaçión delos yndios dela prouinçia de Mechuacán, ms. IV. ç. 5 de la Real Biblioteca de El Escorial. El texto que he utilizado en esta ocasión, y que incluyen los bancos de datos de CORDEy Corpus del español, es el de la edición de 1989 de esta crónica: Relación de Michoacán, ed., introd. y notas de Leoncio Cabrera, Historia 16, Madrid.

${ }^{2}$ Empleo el término americanismo con el sentido de neologismo léxico de América y con base patrimonial hispánica, frente al indigenismo, de origen indígena; si bien, como es sabido, americanismo se emplea por antonomasia para la voz propia de América.

${ }^{3}$ La americanización del léxico es tema recurrente en la obra de los más reconocidos filólogos americanistas, como P. Henríquez Ureña, A. Rosenblat, M.A. Morinigo, G. Friederici, M. Alvar, J.M. Lope Blanch, J.A. Frago Gracia, entre otros. Véanse los trabajos más recientes, con su bibliografía, de A. Torres Torres, Procesos de americanización del léxico hispánico, Universidad, Valencia, 2004 y J.M. ENGUITA UTrill.A, Para la historia de los americanismos léxicos, P. Lang, Frankfurt/M., 2004.
} 
vitalidad en el español internacional a cuenta de su cambio semántico, según se comprueba en los diccionarios, los materiales dialectales $y$, entre ellos, los datos que proporciona el proyecto Varilex ${ }^{4}$. Y creo que, justamente a causa de la vitalidad que logró con tal cambio, su condición primera americana ha pasado inadvertida para los estudiosos del léxico del español de América.

El de camiseta es un caso semejante al de otras palabras del primer español de América, más fácilmente identificables como divergentes del español peninsular, del tipo bezote, gallinazo o tigrillo. Como se sabe, el uso de los sufijos, al margen de su valor propiamente diminutivo, aumentativo o apreciativo, fue muy productivo para dar nombre a lo que requería ser nombrado en América. La creación morfológica por derivación cumplió allí la función de americanizar el léxico patrimonial, renovado así para designar las nuevas cosas, animales, vegetales, minerales o productos. Señala Monge que se trató de "una suerte de formación fabricada (y al mismo tiempo espontánea) con sufijos vivos en la lengua general, y las palabras resultantes fueron, claro está, adultas desde su nacimiento"5.

En un primer momento, lo que me planteé es que la palabra habría viajado con los emigrantes a las Indias. Es lógico considerar primero que camiseta fuera voz traída de España, con una posible procedencia andaluza quizás. E incluso, cabría especular en torno al hecho de que tuviera un origen oriental, por ser filiación dialectal frecuente del sufijo -ete, -eta ${ }^{6}$. Sin embargo, no hay evidencias escritas de que la palabra la llevaran los españoles a las Indias. Antes bien, lo que muestran los documentos coloniales de todo tipo, tanto los literarios como los que tienen un carácter menos cuidado, es que la palabra se empezó a emplear en América. Y hasta el momento, no he hallado ningún testimonio de camiseta con un significado de "vestimenta' que no tenga localización americana, ni anterior al siglo XVI, ni desde este siglo hasta la segunda mitad del siglo XIX.

La acepción actual de camiseta-de la que tratamos en el epígrafe siguiente- es bastante moderna, y con ella ha adquirido la palabra gran vitalidad, siendo más antiguas otras voces también derivadas de camisa y pertenecientes a la misma familia léxica, como camisote, camisola o camisón, datadas desde época medieval.

${ }^{4}$ Proyecto de Variación léxica del español en el mundo, véase http://gamp.c.u-tokyo. ac.jp/ ueda/varilex/index.html (8 de julio de 2010).

${ }^{5}$ FÉLIX Monge, "Aspectos de la sufijación en español", REL, 26 (1996), p. 47.

${ }^{6}$ Véase Fernando GonzÁlez OlLÊ, Los sufijos diminutivos en castellano medieval, CSIC, Anejo LXXV de la RFE, Madrid, 1962, pp. 309-312; Manuel Alvar y BerNARd Pottier, Morfología histórica del español, Gredos, Madrid, 1983, pp. 371-372 y Fernando A. LÁzaro Mora, "Los derivados sustantivos con-ete, -eta", BRAE, 61 (1981), 481-496. 
El $D R A E^{7}$ recoge la variación semántica de camiseta, que sólo se entiende a la luz de su historia, como se verificará más adelante. La edición vigente presenta la siguiente definición:

camiseta. 1.f. Camisa corta y con mangas anchas. 2. f. Prenda interior o deportiva que cubre el tronco, generalmente sin cuello.

La acepción 1 es bastante inespecífica pues, que yo sepa, no se corresponde con el significado actual de la voz camiseta en ningún dialecto hispánico. Como se verá, el diccionario académico reproduce en ella una significación de su propia tradición lexicográfica, la que corresponde al americanismo tratado aquí. Por ello, en mi opinión, el diccionario académico debería adecuar la definición de la entrada camiseta a su significado actual y eliminar esa acepción histórica, de forma que sea el Nuevo diccionario histórico de la lengua española ${ }^{8}$ el que se ocupe de dar cuenta de la historia léxica de camiseta.

En otros diccionarios encontramos definiciones más adecuadas a la prenda actual $1^{9}$. Así, por ejemplo, el diccionario de María Moliner define camiseta como "prenda de punto, de hombre o mujer, con o sin mangas, que se lleva pegada al cuerpo. Algunos tipos se usan como ropa interior y otros, como prenda exterior"10. El Diccionario del espanol actual ${ }^{11}$ de M. Seco et al., por su parte, distingue dos acepciones:

camiseta. 1. Prenda interior, normalmente de punto, sin cuello y con o sin mangas, que cubre el torso. 2. Prenda exterior de punto, con manga corta o sin mangas, y de forma semejante a la camiseta [1] [...] b) Camiseta utilizada en la práctica de algunos deportes, cuyo color representa generalmente el equipo respectivo.

7 Diccionario de la lengua española, 22a ed., Espasa-Calpe, Madrid, 2001.

8 Véase la información sobre el proyecto del nuevo diccionario histórico en la página web de la Real Academia Española, http://www.rae.es/rae.html, dentro de las Obras académicas, así como en J.A. PAScual y R. GARCía Pérez, Limites y horizontes en un diccionario histórico, Diputación, Salamanca, 2007; J.A. Pascual y R. GarCía Pérez, "Estado del Nuevo diccionario histórico de la Real Academia Española", en Diccionario histórico: nuevas perspectivas lingüisticas, ed. M. P. Garcés Gómez, IberoamericanaVervuert, Madrid-Frankfurt/M., 2008, pp. 11-15, y J.A. Pascual y C. Domínguez, "Un corpus para un Nuevo diccionario histórico del español", en Diacronia de las lenguas iberorrománicas: nuevas aportaciones desde la lingüística de corpus, ed. A. EnriqueArias, Iberoamericana-Vervuert, Madrid-Frankfurt/M., 2009, pp. 79-94.

${ }^{9} \mathrm{Al}$ término de esta nota, no ha sido posible la consulta del inminente Diccionario de americanismos académico.

10 María Moliner, Diccionario de uso del español, $2^{a}$ ed., Gredos, Madrid, 1999.

11 Manuel Seco, Olimpia Andrés y Gabino Ramos, Diccionario del español actual, Aguilar, Madrid, 1999. 
Y, por ejemplo, en el Diccionario del español usual en México ${ }^{12}$, dirigido por L.F. Lara, podemos encontrar los dos sentidos con los que se emplea la voz camiseta en los artículos correspondientes a las entradas de las palabras camiseta y playera $^{\mathrm{I3}}$ :

camiseta. 1 Prenda de vestir de algodón u otra tela ligera, sin cuello y con o sin mangas, cerrada y por lo general ajustada, con la que se cubre el tórax. Se usa como ropa interior, aunque hay también especiales para llevarse como prenda exterior, como las que se usan para practicar algunos deportes...

playera. s f Camiseta de algodón, generalmente de manga corta y sin cuello.

\section{EL AMERICANISMO CAMISETA EN LA RELACIÓN DE MICHOACÁN}

Varias razones me llevaron a pensar que camiseta era un americanismo en la Relación de Michoacán (en adelante, $R M$ ). En primer lugar, el hecho de que camiseta apareciera como traducción o equivalente de una voz en la otra lengua, en este caso la lengua tarasca o purépecha: "aquel sacerdote mayor llamado Petámuti... Vestíase una camiseta llamada ucata-tararenguequa negra, y poníase al cuello unas tenazillas de oro y una guirnalda de hilo en la cabeza y un plumaje en un tranzado que tenía como mujer" (p. 38) y "unas camisetas llamadas ucata-tararenguequa y unas guirnaldas de hilo" (p. 74). La RM, como se sabe, es una crónica cuyo texto es la traducción literal al castellano de un relato oral de la historia y de las costumbres del pueblo tarasco, hecho por hablantes de esta lengua. Por consiguiente, el tipo textual de la crónica favorece la creación léxica por las necesidades neológicas que exige la traducción en general ${ }^{14}$. En este sentido, como se aprecia en esos dos contextos, la palabra camiseta funciona como un préstamo semántico ${ }^{15}$.

En segundo lugar, la palabra camiseta está coordinada a la palabra manta; es decir, aparece relacionada con algo de Europa que era tam-

12 Luis Fernando Lara, Diccionario del español usual en México, http://mezcal. colmex.mx/Scripts/Dem/principal.htm ( 8 de julio de 2010).

${ }^{13}$ En Wikipedia, s.v. "camiseta", aparecen las siguientes variantes léxicas por países: camiseta (México, El Salvador, España, Colombia, Costa Rica, Ecuador), remera (Argentina, Paraguay, Uruguay), camisola (Nicaragua), chema (Costa Rica), playera (México, Guatemala, Honduras), polera (Chile, Bolivia), polo (Perú), sueter (Panamá) y franela (Venezuela).

${ }^{14}$ Véase E. Hernández, "Los textos misioneros y la americanización del léxico hispánico: la Relación de Michoacän", en Contactos lingüisticos y culturales en la Nueva España, eds. P. Máynez, S. Equiguas y F. Villavicencio, UNAM, México, en prensa.

15 Sigo la terminología de Valentín García Yebra, "El neologismo", Tradução e Comunicação, 1985, núm. 7, 21-34. 
bién conocido por los españoles: "trujéronles sendas camisetas y otras mantas para ellos y sus mujeres" (p. 83), "algunas mantas y camisetas que llevéis vestidas" (p. 92), "sacaron mantas y camisetas" (p. 166), "y dábanles a cada uno una manta blanca que se cubriesen y otra camiseta colorada, que se vestiese cada uno" (p. 181), "toma estas mantas, y estas cotaras, y este plumaje para la cabeza, y esta camiseta que te pongas y este cincho y petate" (p. 186), "hacíanles dar mantas a los mensajeros y camisetas" (p. 202) y "llevaba mantas para su esposo y camisetas y hachas para la leña de los cúes" (p. 227). En la documentación histórica se verifica que la asociación de manta y camiseta es recurrente en el primer español americano, como se puede comprobar en los testimonios de los bancos de datos de CORDE, Corpus del español o Léxico hispanoamericano ${ }^{16}$. En el uso neológico americano de manta se produce una renovación de su sentido al denotar un objeto indígena, pero en el caso de la palabra camiseta se da un proceso de lexicalización. Así, se forma una nueva palabra mediante la adición del sufijo -ete, -eta a la base léxica camisa, con el que adquiere un sentido independiente. Añadir un sufijo a la realidad europea que les parecía más próxima o semejante fue un procedimiento empleado con frecuencia por los españoles para nombrar las nuevas realidades o conceptos de América. De esta manera, y mediante el procedimiento de la analogía, se reutilizan dos viejas palabras patrimoniales, manta y camiseta, para designar cosas nuevas de América. La reduplicación léxica es habitual en los procesos de americanización del vocabulario del primer español del Nuevo Mundo. Incluso, la voz camiseta llega a aparecer definida o identificada con la palabra manta ("las camisetas que traen, no son sino mantas de mujeres y sayas", p. 94). Y se puede comprobar además, por otro lado, que en casi todos los contextos se encuentran acompañadas de otros préstamos, entre ellos los indigenismos cotara y naguas: "pusiéronle unas naguas muy buenas y otra camiseta encima, y pusiéronle una guirnalda de trébol en la cabeza" (p. 252), "cotaras y camisetas que había tenido cuando pequeño porque esta costumbre era entrellos" (p. 295). Puede el propio contexto proporcionar, por último, el matiz americanizante, dado que siempre el cronista atribuye la vestimenta a los indios: "tenía vestida una camisetay un tranzado de pluma" (p. 77), "debajo de las camisetas que usa esta gente ${ }^{17 "}$ (p. 104), ¿Qué tienes vestido?...una camiseta gorda" (pp. 104-105).

${ }^{16} C O R D E=$ Real Academia Española, Banco de datos (CORDE) [en línea], Corpus diacrónico del español, http://www.rae.es (8 de julio de 2010); Corpus del español= M. Davies, Corpus del español [en línea] http://www.corpusdelespanol.org (8 de julio de 2010); P. Boyd-Bowman, Léxico hispanoamericano (1493-1993), eds. R. Harris-Northall y J. Nitti, Hispanic Seminary of Medieval Studies, 2003, CD-ROM.

17 En esta cita in extenso hay un error de lectura ("teníanlo por gran honra andar así tiznados y, para estar más lucios, y que se les pegase mejor aquel color negro, echaban unas teas en unos braseros, y poníanlas debajo de las camisetas que usa 
Los primeros testimonios de camiseta lexicalizado son tempranos; los hallamos en el Léxico hispanoamericano: " 400 indios con camisetas de librea" [1533 Cuzco], "siete cargas de camisetas para esclavos" [1533 México] y "traen unas camisetas que les llega hasta la rodilla" [1535 México]. En estas documentaciones tiene el significado de 'camisa de indio', localizadas tempranamente tanto en México como en Perú, lo que indica que la voz se expandió con rapidez a través de la lengua de los conquistadores o de los colonizadores por la América hispana; pero, además, entre otras localizaciones, hay documentaciones coloniales tempranas de Ecuador, Colombia, Chile, Guatemala.

No obstante, el primer testimonio del vocablo camiseta corresponde a la Agricultura de Gabriel Alonso de Herrera de 1513 en España ${ }^{18}$, que lo emplea para 'envoltorio de fruto' en: "las auellanas... Si las comen con aquella camiseta que tienen dentro", según CORDE. Sin pretender entrar en la historia léxica de la voz camisa, antecedentes de esta acepción constan dos documentaciones medievales de camisa que no es 'vestido', sino algo relacionado con el mundo vegetal, como camisa de sauco en el Libro de la caza ("en el çumo que sale dela camisa del sauco") y el Libro de los caballos ("hun manoio de la camisa del sauco"), según se comprueba en Corpus del español. En este sentido, Herrera hace uso de varios diminutivos, a partir de camisa, para designar el envoltorio que recubre algunos frutos. En CORDE se comprueba también que el mismo Herrera habla de la "camisilla" de las avellanas y de la "camisita" o película que se forma en el vino. Eso demuestra que camiseta en Herrera es un diminutivo no lexicalizado, y por tanto de interés sólo morfológico, no léxico, en opinión de Pedro Alvarez de Miranda ${ }^{19}$. Esta misma acepción de 'envoltorio' la emplea Fernández de Oviedo en su Batallas y quinquagenas, donde escribía "aquella piedra, enfundada en la camiseta del breuiario", según los materiales de CORDE. En cambio, en su Historia natural de las Indias (ca. 1535), documenta el americanismo: "estaba vestido de una camiseta de algodón".

esta gente, como maredillos, y aquel humo con el calor pegábaseles en el cuerpo y después estregábanse y parábanse muy lucios" [p. 104]), pues en el original se lee maderillos, no maredillos, por lo que sería conveniente corregir el texto en todos los lugares en donde está colgada esta crónica (CORDE, Corpus del español, www. colmich.es).

${ }_{18}$ Por su parte, Corominas, DCEC, s.z. "camisa" sólo da la información de camiseta de su fecha de datación de 1513, la que tomó de $D H$.

19 Debo este dato al profesor y académico Pedro Álvarez de Miranda a quien agradezco su amable atención en la ponencia sobre la crónica de la $R M$ que presenté en el VIII Congreso internacional de historia de la lengua española, Santiago de Compostela 14-18 de septiembre de 2009. Álvarez de Miranda se tomó la molestia de comprobar el dato de Herrera, lo que me ha permitido confirmar con más seguridad mi hipótesis sobre la condición americana de camiseta. 
En el Diccionario histórico $(D H)^{20}$ publicado en 1936 aparecía como primera significación "camisa corta y con mangas anchas" y la autoridad que la sustentaba era Fernández de Oviedo en su Historia natural de Indias: "vieron gente vestida de algodón con mantas delgadas e blancas... e también con camisetas de colores". El segundo testimonio corresponde a Bartolomé de las Casas, en su Apologética historia de Indias: "salían todos aquellos caballeros orejones muy ricamente vestidos con mantas y camisetas ricas de argentería". La tercera es de Gutiérrez Santa Clara, de la Historia de las guerras civiles de Perú y la cuarta de Ovalle, de la Historia de Chile. En CORDE y Corpus del espanol la mayor parte de las documentaciones del siglo xvi corresponden al Perú. Emplean camiseta, entre otros autores, Juan de Betanzos, Diego Trujillo, Pedro Pizarro, Pedro Sarmiento de Gamboa, Juan de Castellanos $^{21}$, José de Acosta y Guamán Poma de Ayala. Por ejemplo, la encontramos en los Comentarios reales del Inca Garcilaso: "el vestido era una camiseta que descendía hasta las rodillas, que llaman uncu" 22 .

Así pues, la palabra viajó pronto y rápido por América; se documenta en 1547 en una carta de Gonzalo Pizarro al gobernador Pedro de Valdivia de Chile: "venía vestido con una camiseta de indios por no ser conocido"; $y$, durante toda la época colonial, la palabra camiseta posee ese sentido de "camisa de indios" en todos los textos de América. Incluso, en 1843, la documentaba Ferrer del Río, en un texto español, la Historia del reinado de Carlos III en España, todavía como traducción al vocablo quechua equivalente: "De su piedad acrisolada ofrecía Macanaz pruebas relevantes, clamando contra terciopelo galoneado de oro vestía, y encima la camiseta o unco de los indios, cabriolé de grana, sombrero de tres picos", según Corpus del español.

En cuanto a la segunda acepción, "camisa corta, ajustada y sin cuello, de franela, algodón o seda, ordinariamente de punto, y que por lo común se pone a raíz de la carne", la atestigua el duque de Rivas en 1854, según $D H$. En $C O R D E$ la documentación primera de esta acepción corresponde a Rosalía de Castro, en La hija del mar 1859: "[la niña] enjugando con la manga de su camiseta una lágrima que rodó silenciosamente por sus tostadas mejillas".

En el cuadro siguiente se expone una síntesis de las documentaciones de las distintas acepciones en CORDE.

${ }^{20} \mathrm{DH}=$ Real Academia Española, Diccionario histórico de la lengua española, Academia Española, Madrid, 1960.

${ }^{21}$ Pasó inadvertida a mi maestro Manuel Alvar, debido a que el contexto en que aparece la voz camiseta en las Elegías de varones ilustres de Juan de Castellanos no apunta, desde luego, maneras de americanismo: MANUEL Alvar, Juan de Castellanos. Tradición española y realidad americana, Instituto Caro y Cuervo, Bogotá, 1972.

22 No aparece en los documentos lingüísticos peruanos, editados y estudiados por José Luis Rivarola, a quien agradezco la amabilidad de permitirme consultar el manuscrito antes de su publicación: J.L. Rivarola, Documentos lingüísticos del Perí. Siglos XVI y XVII. Edición y comentario, CSIC, Madrid, 2009. 


\begin{tabular}{|c|c|c|c|c|c|}
\hline Años & $N^{o}$ de ocurrencias & 'envoltorio' & $\begin{array}{c}\text { 'camisa } \\
\text { de indios' }\end{array}$ & $\begin{array}{c}\text { 'pedazo } \\
\text { de lona' }\end{array}$ & $\begin{array}{c}\text { 'camisa pegada } \\
\text { al cuerpo' }\end{array}$ \\
\hline $1500-1600$ & 51 & 3 & 48 & & \\
\hline $1600-1700$ & 73 & & 73 & & \\
\hline $1700-1800$ & 14 & & 14 & & \\
\hline $1800-1850$ & 6 & & 4 & 2 & \\
\hline $1850-1900$ & 37 & & 1 & & 36 \\
\hline $1900-1950$ & 77 & & & & 77 \\
\hline $1950-2000$ & 137 & & & & 137 \\
\hline
\end{tabular}

Según se aprecia en el cuadro, el cambio semántico tiene lugar en la segunda mitad del siglo XIX. Desde esa fecha hasta la actualidad sólo un testimonio tiene el sentido de 'camisa de indios', mientras que el resto de las documentaciones ya presenta la nueva significación, que está localizada en España también. Hay que advertir que en todos los siglos hay ocurrencias que tienen la marca "España" en CORDE, pero se trata de documentaciones de textos americanos, que corresponden a obras de cronistas o de historiografía americana, aunque estén publicadas en España (de Fernández de Oviedo, José de Acosta, Jiménez de la Espada, entre otros).

Respecto de otros derivados de la misma familia léxica, hay documentaciones más antiguas. Así, en los materiales inéditos del Diccionario de la lengua castellana hasta finales del siglo $X \mathrm{~V}^{23}$, del malogrado proyecto lexicográfico de la sección de Filologia del antiguo Centro de Estudios Históricos, no se documenta camiseta, pero sí los derivados castellanos camisote y camisón, así como las voces camisot, camitsery camisallo de Inventarios aragoneses del siglo XIV. Entre los materiales de este diccionario medieval hay documentaciones de camisón que datan de 1399 y del Cancionero de Alfonso de Baena. En CORDE hay documentaciones antiguas de camisate. Una es la del anónimo de Las siete partidas de Alfonso $X$, de 1491, en donde se describe la prenda como "lorigon es dicho aquel que lieua la manga fasta al cobdo \& no passa mas adelante fasta la mano \& camisote es el que llega la manga fasta la mano E guardabraços es el que tiene mangas". De todas ellas se pueden consultar documentaciones en $D C E H$, así como en los tesoros lexicográficos (Tesoro, de Gili Gaya, $N T L E$ y $N T L L E^{24}$ ). Pero lo que

${ }^{23}$ El fichero del "Diccionario de la lengua castellana hasta finales del siglo xv", que contiene los materiales de trabajo, sin elaborar, del diccionario histórico medieval inédito, que fue dirigido por Américo Castro (Memoria correspondiente a los años 1914 y 1915, Junta para Ampliación de Estudios e Investigaciones Científicas, Madrid, 1916, pp. 168-169), se encuentra depositado en el Instituto de Lengua, Literatura y Antropología, del Centro de Ciencias Humanas y Sociales del CSIC.

${ }_{24}$ DECH = J. Corominas y J.A. PASCUAL, Diccionario crítico etimológico castellano e hispánico, Gredos, Madrid, 1980-1991; Tesoro= S. GILI GAYA, Tesoro lexicográfico 
interesa ahora es destacar el hecho de que el sufijo -ete, -eta estaba disponible para la lexicalización. Estructuralmente había una casilla vacía en la familia léxica de camisa para formar una nueva palabra que significara o diera nombre a la prenda indígena mediante la adición de ese sufijo. En la definición de $D R A E$ se dice que el sufijo se emplea "para formar diminutivos, despectivos u otras palabras de valor afectivo, a veces de manera no muy explícita, a partir de adjetivos y sustantivos... Muchas palabras han perdido estos valores. Peseta, juguete...”.

Por otro lado, como el sufijo -ete, -eta tiene una marcada filiación dialectal consideré la posibilidad de que el cronista anónimo de la $R M$ tuviera una procedencia oriental; es decir, un origen aragonés o catalán, o de las hablas meridionales de su influencia. Pharies ${ }^{25}$, sin embargo, nos previene de que este sufijo no marca de modo seguro la procedencia de algunos derivados, pues señala que:

no hay duda de que -ete, -eta se ha hecho productivo en castellano, pero no siempre resulta fácil identificar los derivados propios, pues muchos tienen equivalentes catalanes, cf. caballete 'caballo pequeño', 'potro de madera' [1430] (cavallet), barrilete 'vasija de aceite' [1490] (barrilet), camiseta 'camisa corta y con mangas anchas' [1513] (camiseta) pileta 'pila pequeña de iglesia' [1513 caleta 'ensenada pequeña' [1535] (caleta) y rodete 'rosca de pelo o de lienzo' [xvi] (rodet).

Pero Pharies utiliza la datación de 1513 para camiseta, probablemente a partir del $D H$ o del $D C E H$, la que, como hemos visto, no corresponde con la acepción de la 'vestimenta', sino con la de 'envoltorio'. Y se comprueba que, en catalán, tampoco hay dataciones de camiseta antiguas, anteriores al siglo XVı, ni en el Diccionario catalán de Corominas ni en el de Alcover y Moll; por otro lado, tampoco aparece en el Corpus Informatitzat del Català Antics (CICA) ${ }^{26}$. Por consiguiente, hay que descartar una procedencia dialectal relacionada con las lenguas o hablas del oriente de la Península.

(1492-1726), Instituto A. de Nebrija-CSIC, Madrid, 1960 [t. 1: A-E]. NTLE = Nuevo tesoro lexicográfico del español (siglo XIV-1726), dirs. L. Nieto Jiménez y M. Alvar Ezquerra, Real Academia Española-Arco Libros, Madrid, 2007. NTLLE = Real Academia Española, Nuevo tesoro lexicográfico de la lengua española, Espasa Calpe, Madrid, 2001 (edición en DVD).

${ }_{25}$ R. Pharies, Diccionario etimológico de los sufijos españoles y de otros elementos finales, Gredos, Madrid, 2002.

${ }^{26}$ J. Corominas, Diccionari etimologic i complementari de la llengua catalana, Curial Edicions Catalanes, Barcelona, 1980-1991; AnTONi MARIA Alcover y Francesc de B. MolL, Diccionari català-valencià-balear, Editorial Moll, Palma de Mallorca, 19641969; Corpus informatitzat del Català antic (CICA), dir. J. Torruella, junt amb Manel Pérez Saldanya i Josep Martines. http://lexicon.uab.cat/cica. 2009 (8 de julio de 2010). Agradezco la amabilidad de la profesora Gloria Clavería por permitirme consultar su base de datos. 
Cabría considerar, asimismo, que camiseta fuera un andalucismo léxico, pero ni hemos encontrado hasta el momento ninguna prueba documental, ni, desde el siglo XVI hasta el XIX, camiseta aparece en contextos que no sean americanos. Por consiguiente, en tanto no aparezca algún testimonio anterior al descubrimiento de América, es voz de creación americana, al no atestiguarse la acepción de vestimenta en ningún documento peninsular ${ }^{27}$ hasta la segunda mitad del siglo XIX, momento en que la palabra adquiere una nueva vida con otro sentido.

En verdad, sucede que la acepción de la palabra camiseta que hoy conocemos es moderna, lo mismo que lo es la realidad a la que representa, frente a las prendas y voces propias de las hablas populares camisa y camisón, según se puede comprobar en los atlas lingüísticos españoles ( $A L E A, V$, mapa 1316, 1387, ALEICan, II, mapa 536) ${ }^{28}$. Y un claro ejemplo de ello es que camiseta no la registró el primer diccionario monolingüe del español, es decir, el Tesoro de la lengua castellana de Sebastián de Covarrubias ${ }^{29}$. Por otro lado, es lástima que el Atlas lingüistico de Hispanoamérica no llegara a publicarse cartográficamente ni en su integridad, pues constituía la pregunta 101 de su Cuestionario ${ }^{30}$.

\section{HISTORIA LEXICOGRÁFICA DE CAMISETA}

El primer diccionario académico incluyó camiseta y proporcionó dos autoridades americanas, el Inca Garcilaso y Ovalle:

camiseta. s.f. dim. de camisa. La camisa corta, y con mangas anchas. Lat. Parvum Indufium Subucula. Inca. Coment. R. lib. I, cap. 5. Tenian camisetas, y mangas de algodón, blancas y de colores. Ov. Hist. de Chile, fol. 91. El cuerpo le cubren con la que llamámos camiseta, y ellos Macun, que va tambien inmediata.

Desde su primera edición de Autoridades hasta la edición de 1817, la Academia no introduce ninguna modificación en la definición;

${ }^{27}$ Creo significativa que no aparezca en la documentada tesis doctoral, dirigida por J.A. Frago Gracia, de Mariano Franco Frgueroa, Léxico hispanoamericano de los siglos XVI y XVII en fuentes de América Central y de la Nueva España, Universidad, Sevilla, 1991.

28 ALEA = Manuel Alvar, con la colaboración de Antonio Llorente y Gregorio Salvador, Atlas lingüístico y etnográfico de Andalucia, Universidad-CSIC, Granada, 1961-1973; ALEICan = MANUEL ALvAR, Atlas lingüistico y etnográfico de las Islas Canarias, Cabildo Insular, Gran Canaria, 1975-1978.

${ }^{29}$ Sebastián De Covarrubias, Tesoro de la lengua castellana o española [Madrid, 1611], ed., introd. e índice de Martín de Riquer, Barcelona, 1987.

${ }_{30}$ Manuel Alvar y Antonio Quilis, Atlas lingüístico de Hispanoamérica. Cuestionario, est. introd. de Manuel Alvar, Instituto de Cooperación Iberoamericana, Madrid, 1984. 
en ésa le asigna la marca de voz anticuada y una matización de quien la viste: "camiseta: $s$. f. ant. La camisa corta y con mangas anchas, hoy tiene uso para denotar el vestido exterior de las mugeres", según NTLLE. Ahora bien, otros lexicógrafos sí supieron percibir su condición americana y quien primero entiende camiseta como voz provincial, localizada además en el Perú, fue Salvá en 1846: "Camisa de tela ordinaria de lana que usan los trabajadores y gente pobre del Perú". Esta especificación es reproducida por otros lexicógrafos, como Gaspar en 1853 y Domínguez en 1859. Sustancialmente mejorada la registra en 1917 Alemany, quien reúne las otras acepciones pero además da por vez primera la marca de América. Define camiseta del siguiente modo: "En Bolivia, camisón blanco sin mangas y ceñido que usan algunos indios”. En 1852, Castro, además de la definición primera académica, da cuenta del uso de camiseta como término marinero, definido como "Pedazo de lona ó vitre con que se cubren y sujetan algunas velas después de aferradas". Fue en la edición de 1884 cuando la Academia perdió la marca de voz anticuada, que ya no recuperará. Y en la edición siguiente, de 1899, introduce el nuevo sentido de la palabra, sin eliminar la primera acepción: "Camisa corta, ajustada y sin cuello, de franela, algodón ó seda, ordinariamente de punto y que por lo común se pone á raíz de la carne". En 1927, la Academia presenta una ilustración de camisetay, en la edición primera de 1936, da a las acepciones sus correspondientes autoridades. A partir de la segunda edición de 1936, las ediciones sucesivas proporcionan la definición de la edición vigente que veíamos arriba.

En NTLE aparece el diccionario bilingüe que primero registró camiseta, el hispano-inglés de Minsheu de 1599: "camiceta, vide camiseta... a little shirt or smock...”. Pero, como es lógico, son los vocabularios hispano-amerindios los primeros que la recogen. Primero lo hizo el anónimo vocabulario hispano-quechua, más conocido por el nombre de su impresor, Antonio Ricardo, en 1586, que reúne tres entradas ${ }^{31}$ :

Camiseta de indio. vncucusma (Chin).

Camiseta axedrezada. cassana vncu.

Camiseta ponerse. vncu llicuni.

A él le siguió el vocabulario quechua de González Holguín ${ }^{32}$, quien reúne varias entradas en torno a este concepto en su parte castellano-quechua:

31 Anónimo [= Antonio Ricardo], Arte, y vocabulario en la lengva general del Perv llamada Quichua, y en la lengua Española. El más copioso y elegante que hasta agora se ha impreso. En los Reyes. Por Antonio Ricardo. Año de M.D.LXXXVI, Antonio Ricardo, Lima, 1586.

32 Diego González Holguín, Vocabvlario de la lengva general de todo el Perv llamada lengua qquichua, o del Inca, Francisco del Canto, Lima, 1608. 
Camiseta de indio. Vncu.

Camiseta axedrezada en los ombros y triangulada. Ahuaqui cassana vncu. Camiseta ponerse. Vncullicuni, desnudarla, chutucuni vncucta, o llusticuni o llatanacuni.

Vestidura larga que arrastra. Cuyzuppacha, y uncuçapa el de la larga camiseta.

Vestirse la camiseta el yndio. Vncullicuni. Y la manta. Yacollacuni.

$\mathrm{Y}$, en la parte del diccionario que empieza por la lengua indígena, aparecen las siguientes entradas:

Ahuaquivncu. La camiseta axedrezada de los hombros al pecho.

Cuchu vncu. Camiseta de colores con labores galana.

Ccori, o collqque uncu. Camiseta chapeada de oro, o de plata.

Cсисси сcucculla, o sumpu sumpulla vncu. Camiseta, o cosa angosta estrecha.

Kassana uncu. Camiseta axedrezada de cumbi.

Huampar vncu. Camiseta de pinturas triangulares.

Ticlla. Cosa hecha de los dos colores blanco de vna haz y negro de otra como camiseta.

Vncu. Camiseta de indios.

Iñaca acsu, o yñacalliclla. Vestido de muger galan pintado de lauores. Para vestido galan de varones ttupa ppachha, o incappachha ttupa vncu inca vncu. Camiseta galana.

Los vocabularios de las lenguas indígenas con el español son unas fuentes útiles para el conocimiento del español americano en el nivel léxico, especialmente en lo que afecta a los distintos procesos neológicos a que da lugar el contacto entre lenguas y culturas, según muestran los vocabularios del quechua en el caso que se ha tratado aquí de la palabra camiseta ${ }^{33}$.

\section{ConcLusión}

La historia de las palabras normalmente coincide con la historia de las cosas y, en este caso, hemos visto cómo nace una palabra para designar una cosa nueva de América, pero también cómo se pierde su uso por la decadencia del objeto al que nombra. La documentación existente indica que la palabra no vino de España, sino que se formó en América mediante recursos internos de la lengua -y habituales en los procesos de americanización del léxico-, para designar

33 La catalogación y el estudio de los vocabularios hispano-amerindios de la etapa colonial son los objetivos prioritarios del proyecto "FFr08-03457: Corpus de vocabularios bilingües iberoamericanos (siglos XVI-XVIII). Segunda etapa", financiado por la Dirección General de Investigación del Ministerio de Ciencia e Innovación de España, en el que se ha enmarcado el presente trabajo. 
una prenda de la indumentaria indígena. Durante la época colonial la población de habla española empleó camiseta para referirse a la prenda indígena en toda América y también lo hicieron quienes escribían sobre América y publicaban en España. Pero andando el tiempo, seguramente porque la cosa o la prenda va dejando de existir, la lengua renueva su significado. $Y$ la vitalidad que adquiere el nuevo sentido de la palabra camiseta ensombrece su primer uso, lo que ha hecho que su condición americana haya pasado desapercibida a los estudiosos del español de América.

Propongo que en la definición de camiseta del diccionario académico se elimine la primera acepción, pues es histórica y no representa ningún uso del español actual. $\mathrm{O}$ bien, si se prefiere, que se introduzca, o mejor dicho recupere la marca de "voz anticuada"34, y, de paso, se incluya la marca geográfica de "voz de América".

ESTHER HERNÁNDEZ Instituto de Lengua, Literatura y Antropología, CCHS-CSIC

${ }^{34}$ Opción más acorde con los procedimientos de actuación académicos, puesto que en el prólogo de su vigésima edición se dice que "el repertorio académico, en tanto no se haya completado la redacción del Diccionario histórico, debe seguir albergando una selección del léxico hispánico cronológicamente desfasado, que permita al lector interpretar los textos clásicos de nuestra lengua. Las voces y acepciones cuya vigencia actual, de acuerdo con la documentación académica, no está probada, llevan una marca que las asigna a uno de estos grupos". 